\title{
Physical Exercise Prescription for Individuals with Kidney Disease
}

\section{Getu Teferi", Bayih Ejigu}

Department of Sports Science, Debremarkos University, Debremarkos, Ethiopia

Email address:

teferigetu36@gmail.com (G. Teferi)

${ }^{*}$ Corresponding author

\section{To cite this article:}

Getu Teferi, Bayih Ejigu. Physical Exercise Prescription for Individuals with Kidney Disease. American Journal of Science, Engineering and Technology. Vol. 5, No. 4, 2020, pp. 124-127. doi: 10.11648/j.ajset.20200504.11

Received: August 26, 2020; Accepted: September 9, 2020; Published: November 9, 2020

\begin{abstract}
Chronic kidney disease (CKD) is a common condition that has significant implications for patients' health. CKD is diagnosed if evidence of kidney damage has been present for more than 3 months; it is divided into five stages depending on the glomerular filtration rate (GFR). CKD is asymptomatic and determining its prevalence relies on screening populations, so the reported prevalence depends on the population studied and screening methods used. Risk factors for CKD can be divided into initiating and perpetuating factors, and includes genetic factors, ethnicity, socio-economic factors and age. There are several causes of CKD, the most common being diabetes mellitus. In order to reduce the burden of CKD, it is essential to recognize which patients are at most risks so that they can be screened and treated early. It is hoped that with early recognition and treatment the number of patients with CKD progressing to end-stage kidney disease (ESKD) and the need for renal replacement therapy will be reduced. Exercise testing of individuals with CKD should be supervised by trained medical personnel, with the use of standard test termination criteria and test termination methods. Based on current evidence, exercise is safe for these individuals if performed at moderate intensity and if progression occurs gradually.
\end{abstract}

Keywords: Chronic Kidney Disease, Dialysis, Kidney Transplantation, Exercise Testing, Exercise Prescription

\section{Introduction}

Most people are born with two kidneys, each growing to the size of our fist. Our kidneys are bean shaped and are positioned near the middle of our back, on either side of our backbone (spine). Our kidneys are part of the body's urinary system [1]. Chronic kidney disease, also called chronic kidney failure, describes the gradual loss of kidney function. Our kidneys filter wastes and excess fluids from our blood, which are then excreted in our urine. When chronic kidney disease reaches an advanced stage, dangerous levels of fluid, electrolytes and wastes can build up in our body [2].

Kidney disease affects approximately 26 million American adults. It occurs when our kidneys become damaged and can't perform their function. Kidney disease can lead to other health problems, including weak bones, nerve damage, and malnutrition.

High blood pressure is dangerous for the kidneys because it can increase the pressure on the glomeruli. Glomeruli are the tiny blood vessels in the kidneys where blood is cleaned.
Over time, the increased pressure damages these vessels and kidney function begins to decline. Kidney function will eventually deteriorate to the point where the kidneys can no longer perform their job properly. In this case, a person would need to go on dialysis. Dialysis filters extra fluid and waste out of the blood. Dialysis can help treat kidney disease but it can't cure it. A kidney transplant may be another treatment option depending on your circumstances.

Diabetes is also a major cause of chronic kidney disease. Diabetes is a group of diseases that causes high blood sugar. The increased level of sugar in the blood damages the blood vessels in the kidneys over time. This means the kidneys can't clean the blood properly. Kidney failure can occur when your body becomes overloaded with toxins [3]. If the disease gets worse over time, our kidneys may stop working completely. This means that dialysis will be required to perform the function of the kidneys. Dialysis is a treatment that filters and purifies the blood using a machine. It can't cure kidney disease, but it can prolong your life. 


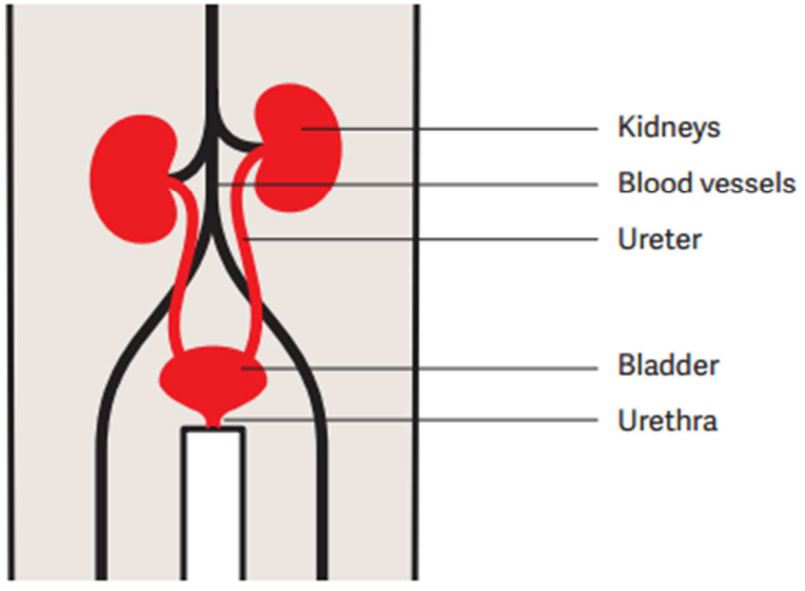

Figure 1. Anatomical location of kidney.
Individuals are diagnosed with CKD if they have kidney damage evidenced by microalbuminuria or have a glomerular filtration rate $<60 \mathrm{~mL} \cdot \mathrm{min}^{-1} .1 .73 \mathrm{~m}^{-2}$ for $>=3 \mathrm{mo}$. Based on National Kidney Foundation's Kidney Disease Outcomes Quality Initiative (K/DOQI) Guidelines, CKD is divided into five stages primarily depending on the glomerular filtration rate of the individual and the presence of kidney damage. Approximately 23 million Americans have CKD, and the prevalence of the disease is estimated to be $11.5 \%$. Hypertension, DM, and CVD are very common in the CKD population with the prevalence of these comorbidities rising incrementally from stage 1 to stage $5 \mathrm{CKD}$. When individuals progress to stage 5 CKD (i.e., glomerular filtration rate $<15$ $\mathrm{mL}$. min-1.1.73 $\mathrm{m}^{-2}$ ) their treatment options include renal replacement therapy (hemodialysis or peritoneal dialysis) or kidney transplantation [4].

Table 1. Glomerular Filtration Rate (GFR) Categories in Chronic Kidney Diseases (13).

\begin{tabular}{lll}
\hline GFR Category & GFR $\left(\mathbf{m L}^{*} \mathbf{m i n}^{-1 *} \mathbf{. 7 3} \mathbf{m}^{-\mathbf{2}}\right.$ & Terms \\
\hline G1 & $\geq 90$ & Normal or high \\
G2 & $60-80$ & Mildly decreased \\
G3a & $45-59$ & Mildly to moderately decreased \\
G3b & $30-44$ & Moderately to severely decreased \\
G4 & $15-29$ & Severely decreased \\
G5 & $<15$ & Kidney failure \\
\hline
\end{tabular}

\section{Functions of Kidneys}

Kidneys are the unsung heroes of the body. The kidneys play a number of important roles in the body such as:

1) Extracting excess fluid to make urine

2) Controlling blood pressure

3) Filtering waste products from the blood

4) Controlling body fluids

5) Stimulating the production of red blood cells

6) Controlling potassium, calcium and phosphorus levels regulating vitamin $\mathrm{D}$

Sometimes kidney function can change quickly. For example, our kidneys may stop working properly because of a sudden loss of large amounts of blood (e.g. during surgery) or as a result of an accident, illness or infection.

A sudden change in kidney function is called acute kidney injury. This is often temporary but can occasionally lead to lasting kidney damage. More often kidney function worsens over a number of years - it is a chronic, or ongoing, condition. Chronic kidney disease (also referred to as CKD) is called a silent disease there are often no warning signs. Sometimes people lose up to 90 per cent of their kidney function before getting any symptoms.

\section{Exercise Testing}

Because CVD is the major cause of death in individuals with CKD, diagnostic exercise testing is indicated. Exercise testing is included in the pre-transplantation workup for kidney recipients [5] However, some authorities believe diagnostic testing for patients with end-stage renal disease (i.e., stage $5 \mathrm{CKD}$ ) is not warranted because their performance on a symptom-limited exercise test is affected by muscle fatigue, and such testing may act as an unnecessary barrier to their participation in a training program [6]. Exercise testing of individuals with CKD should be supervised by trained medical personnel, with the use of standard test termination criteria and test termination methods.

Most research on patients with CKD has been done on individuals classified with stage 5 CKD. Current evidence suggests these individuals tend to have low functional capacities with values that are approximately $60 \%-70 \%$ of those seen in healthy age and sex-matched controls [5]. $\mathrm{V}^{\cdot} \mathrm{O} 2_{\text {peak }}$ ranges between $17-28.6 \mathrm{~mL} \cdot \mathrm{kg}^{-1} \mathrm{~min}^{-1}$ and can be increased with training by approximately $17 \%$ but never reach the values achieved by age and sex-matched controls. This reduced functional capacity is thought to be related to several factors including a sedentary lifestyle, cardiac dysfunction, anemia, and musculoskeletal dysfunction.

The following exercise testing considerations should be noted:

1) Medical clearance should be obtained from both the patient's primary care physician and nephrologist.

2) Individuals with $\mathrm{CKD}$ are likely to be on multiple medications including those that are commonly used in the treatment of hypertension and DM.

3) When performing a graded exercise test on individuals with stage 1-4 CKD, standard testing procedures should be followed. However, in patients receiving maintenance hemodialysis, testing should be scheduled for non-dialysis days and BP should be monitored in the arm that does not contain the arterio-venous fistula [7]. 
4) Patients receiving continuous ambulatory peritoneal dialysis should be tested without dialysate fluid in their abdomen. Standard procedures are used to test patients that are transplant recipients [7].

5) Both treadmill and cycle leg ergometry protocols have been used to test individuals with kidney diseases, with the treadmill being more popular. Because of the low functional capacity in this population, more conservative treadmill protocols such as the modified Bruce protocol, Balke, Naughton, or branching protocols are appropriate. If cycle leg ergometry is used, initial warm-up work rates should be $20-25 \mathrm{~W}$ with the work rate increased by 10 $30 \mathrm{~W}$ increments every $1-3 \mathrm{~min}$ [8].

6) In patients receiving maintenance hemodialysis, peak heart rate $\left(\mathrm{HR}_{\text {peak }}\right)$ is approximately $75 \%$ of agepredicted maximum. Because HR may not always be a reliable indicator of exercise intensity in patients with CKD, RPE should always be monitored.

7) Isotonic strength testing should be done using a 3-RM or higher load (e.g., 10-12-RM) because 1-RM testing is generally thought to be contraindicated in patients with CKD because of the fear of spontaneous avulsion fractures.

8) Muscular strength and endurance can be safely assessed using isokinetic dynamometers employing angular velocities ranging from 60 degrees to 180 degrees. $\mathrm{S}^{-1}$ is recommended.

9) As a result of the very low functional capacities of individuals with $\mathrm{CKD}$, with an estimated $50 \%$ not being able to perform symptom-limited testing, traditional exercise tests may not always yield the most valuable information [9]. Consequently, a variety of physical performance tests that have been used in other populations (e.g., older adults) can be used. Tests can be chosen to assess CRF, muscular strength, balance, and flexibility.

\section{Exercise Prescription}

The ideal FITT principle of Ex Rx for individuals with CKD has not been fully developed, but based on the research that has been done, programs for these patients should consist of a combination of aerobic and resistance [10]. It seems prudent to modify the recommendations for the general population; initially using light-to-moderate intensities and gradually progressing over time based on individual tolerance. Medically cleared recipients of kidney transplants can initiate exercise training as early as $8 \mathrm{~d}$ following the transplant operation [11]

\section{FITT Recommendations for Individuals with Chronic Kidney Disease}

Aerobic, Resistance, and Flexibility Exercise

The following FITT principle of Ex Rx is recommended for individuals with CKD:
Frequency: Aerobic exercise $3-5 \mathrm{~d} . \mathrm{wk}^{-1}$; resistance exercise 2-3 d. $\mathrm{wk}^{-1}$.

Intensity: Moderate intensity, aerobic exercise $(40 \%-<60 \%$ VO2R, RPE 11-13 on a scale of 6-20); resistance exercise, 70\%-75\% 1-RM.

Time: Aerobic exercise 20-60 min of continuous activity; however, if this amount cannot be tolerated, 3-5 min bouts of intermittent exercise aiming to accumulate $20-60$ min. $^{-1}$ is recommended; Resistance training, a minimum of 1 set of $10-15$ repetitions.

Choose 8-10 different exercises to work the major muscle groups. Flexibility exercise should be performed following the guidelines for healthy adults.

Type: Walking, cycling, and swimming are recommended aerobic activities.

Use either machine weights or free weights for resistance exercise.

Special considerations

1) Individuals with CKD should be gradually progressed to a greater exercise volume over time. Depending on the clinical status and functional capacity of the individual, the initial intensity selected for training should be light (i.e., $30 \%-40 \% \mathrm{~V}^{*} \mathrm{O} 2 \mathrm{R}$ ) and for as little as $10-15 \mathrm{~min}$ of continuous activity or whatever amount the individual can tolerate. The duration of the physical activity should be increased by $3-5 \mathrm{~min}$ increments weekly until the individual can complete 30 min of continuous activity before increasing the intensity [2].

2) The clinical status of the individual is important to consider. The progression may need to be slowed if the individual has a medical setback.

3) Some individuals with CKD are unable to do continuous exercise and therefore should perform intermittent exercise with intervals as short as $3 \mathrm{~min}$ interspersed with $3 \mathrm{~min}$ of rest (i.e., 1:1 work-to-rest ratio). As the individual adapts to training, the duration of the work interval can be increased, whereas the rest interval can be decreased. Initially, a total exercise time of 15 min can be used, and this can be increased within tolerance to achieve up 20-60 $\mathrm{min}$ of continuous activity.

4) Individuals with CKD performing resistance exercise should be asked to perform at least 1 set of 10 repetitions at $70 \% 1-\mathrm{RM}$ twice per week. Consider adding a second set when the individual can easily complete 15 repetitions at a specific weight.

Hemodialysis:

1) Exercise can be performed on non-dialysis days and should not be done immediately post-dialysis.

2) If exercise is done during dialysis, exercise should be attempted during the first half of the treatment to avoid hypertensive episodes.

3) Place great emphasis on the RPE because HR is unreliable.

4) Patients may exercise the arm with the arterio-venous access as long as they do not directly rest weight on that 
area. Measure BP in the arm that does not contain the fistula [2].

Peritoneal Dialysis:

Patients on continuous ambulatory peritoneal dialysis may attempt exercising with fluid in their abdomen; however, if this produces discomfort then they should be encouraged to drain the fluid before exercising [12]. Recipients of Kidney Transplants: During periods of rejection, the FITT principle of Ex Rx should be reduced but exercise can still be continued.

\section{Conclusion}

Individuals with $\mathrm{CKD}$ tend to be much reconditioned depending on their age and disease status. Based on current evidence, exercise is safe for these individuals if performed at moderate intensity and if progression occurs gradually. Individuals with CKD should be gradually progressed to a greater exercise volume over time. Exercise testing should be done under medical supervision and may involve the use of functional tests rather than the traditional GXT. Exercise can be performed on non-dialysis days and should not be done immediately post-dialysis. During periods of rejection, the FITT principle of Ex Rx should be reduced but exercise can still be continued.

\section{References}

[1] U.S. Renal Data System. USRDS 2009 Annual Data Report: Atlas of Chronic Kidney Disease and End-Stage Renal Disease $\mathrm{i} \mathrm{n}$ the United States [Internet]. Bethesda (MD): National Institutes of Health, National Institute of Diabetes and Digestive and Kidney Disease; [ci ted 2015 Jan 15]. Available from: http: //www.usrds.org/atl as09.aspx.

[2] Johansen KL. Exercise and chronic kidney disease: current recommendations. Sports Med. 2005; 35 (6): 485-99.

[3] Centers for Disease Control and Prevention. National Chronic
Kidney Disease Fact Sheet: General Information and National Estimates on Chronic Kidney Disease in the United States, 2014. Atlanta (GA): U.S. Department of Health and Human Services, Centers for Disease Control and Prevention; 2014. 4 p.

[4] KDIGO 2012) Kidney Disease: Improving Global Outcomes. KDIGO 2012 Clinical Practice Guideline for the Evaluation and Management of Chronic Kidney Disease. Kidney International Supplements. 2013; 3: 134-5.

[5] Johansen KL, Painter P. Exercise in individuals with CKD. Am J Kidney Dis. 2012; 59 (1): 126-34.

[6] American Association on Intellectual and Developmental Disabilities. Intellectual Disability: Definition, Classification, and Systems of Support. 11th ed. Washington (DC): Ameri can Association on Intellectual and Developmental Disabilities; 2010. 280 p.

[7] Painter PL, Hector L, Ray K, et al. A randomized trial of exercise training after renal transplantation. Transplantation. 2002; 74 (1): 42-8.

[8] Violan MA, Pomes T, Maldonado S, et al. Exercise capacity in hemodialysis and renal transplant patients. Transplant Pr oc. 2002; 34 (1): 417-8.

[9] Diesel W, Noakes TD, Swanepoel C, Lambert M. Isokinetic muscle strength predicts maxi mum exercise tolerance in renal patients on chronic hemodialysis. Am J Kidney Dis. 1990; 16 (2): 109-14

[10] Heiwe S, Jacobson S. Exercise training i n adults with CKD: a systematic review and meta-analysis. Am J Kidney Dis. 2014; 64 (3): 383-93.

[11] Miller TD, Squires R W, Gau GT, Ilstrup DM, Frohnert PP, Sterioff S. Graded exercise testing and training after renaltransplantation: a preliminary study. Mayo ClinPr oc. 1987; 62 (9): 773-7.

[12] Painter PL. Exercise after renal transplantation. Adv Ren Replace Ther. 1999; 6: 159-64.

[13] ACSM's. Guidelines for Exercise Testing and Prescription, $10^{\text {th }}$ ed., Lippincott, Williams and Wilkins, Philadelphia, 2018. 